\title{
Thoracoscopic Sympathectomy for Hyperhidrosis
}

\section{Yasser Abdurabu Obadiel1, Abdullah Mohammed Megally², Mohammed Abdulla Alqatta², Thyazan Almorish ${ }^{3}$, Zahra'a Abdullah Megally3, Hadi Mujlli1}

\author{
${ }^{1}$ Thamar Medical University, Dhamar, Yemen \\ ${ }^{2}$ Sanaa Medical College, Sana'a University, Sanaa, Yemen \\ ${ }^{3}$ Al-Kuwait Teaching Hospital, Sanaa, Yemen \\ Email: drhadimujlli@gmail.com
}

How to cite this paper: Obadiel, Y.A., Megally, A.M., Alqatta, M.A., Almorish, T., Megally, Z.A. and Mujlli, H. (2020) Thoracoscopic Sympathectomy for Hyperhidrosis. Open Access Library Journal, 7: e6864.

https://doi.org/10.4236/oalib.1106864

Received: September 26, 2020

Accepted: November 20, 2020

Published: November 23, 2020

Copyright $\odot 2020$ by author(s) and Open Access Library Inc.

This work is licensed under the Creative Commons Attribution International License (CC BY 4.0).

http://creativecommons.org/licenses/by/4.0/

\begin{abstract}
The aim of the study: The goal of this retrospective study was to evaluate the outcomes of bilateral thoracoscopic sympathectomy for primary hyperhidrosis. Methods. We identified all patients who underwent bilateral thoracoscopic sympathectomy between January 2017 and May 2020, in Sana'a (capital of YEMEN) hospitals. Details of pre-operative symptoms, surgical procedure and post-operative complications were collected from the patient's files, and each patient was sent a questionnaire regarding success of the procedure, and compensatory sweating through their phone number by what Sapp. Results. We had 36 patients complaining of hyperhidrosis: 27 cases were males and 9 cases were females, age range $14-32$ years at the time of surgery. All of them suffered from hyperhidrosis in the palm and sole, 15 cases of them suffered from hyperhidrosis in axilla, and 10 cases of patients were associated with facial blushing. All cases improved from sweat in the palm (100\%), and most of them improved from the sweat of sole (94.6\%). Conclusion: Thoracoscopic sympathectomy is effective in the treatment of hyperhidrosis. However, compensatory hyperhidrosis seems unavoidable complication and infrequently improves with time, so patients should be clearly informed before committing to surgery.
\end{abstract}

\section{Subject Areas}

Surgery \& Surgical Specialties

\section{Keywords}

Sympathectomy, Hyperhidrosis, Sweating, Thoracoscopic

Sympathectomy 


\section{Introduction}

Hyperhidrosis can be defined as a pathologic condition characterized by excessive sweating beyond the organism's physiological needs to maintain the body temperature within an adequate range. Hyperhidrosis is divided into primary or secondary. Primary hyperhidrosis is the pathology itself, characterized by the excessive sweating. The etiology of primary hyperhidrosis remains unknown despite multiple literature reviews [1]. Genetic factors may play a role in excessive neural stimulation, although this is not well understood. It occurs in about $2 \%$ of the world population, affecting equally men and women. The primary hyperhidrosis is usually localized and mostly in palmar, axilla, and/or sole. Craniofacial hyperhidrosis can occur, alone or with facial blushing. Secondary causes are usually easier to identify because they are associated with medications such as dopamine agonists, selective serotonin release inhibitors (SSRIs), antipsychotics, alcohol, and insulin, systemic disorders such as diabetes mellitus, hyperthyroidism, Parkinson disease, and other neurologic disorder, and tumors such as pheochromocytoma and lymphoma [2]. A variety of clinical treatments can be tried to use in primary hyperhidrosis. These treatments are indeed less invasive than a surgical intervention, but all of them have only a palliative potential and, once stopping the therapy, the symptoms will relapse in virtually all patients. Sweat glands ablation by energy-based devices is a promising nonsurgical alternative to treat hyperhidrosis, especially for axillary cases. The approved devices use microwave energy, high-intensity focused ultrasound or laser therapy [3]. From a practical point of view, it means that the majority of patients will eventually end up seeking a surgical consultation. There are several ways to treat excessive sweating: antiperspirants (e.g. Aluminium Zirconium, Tetrachlorohydrex Glycine), iontophoresis, anticholinergic medication, psychotherapy, botulinum toxin injections and surgery. Recently, there is increased awareness of the use of botulinum toxin injections as a non-surgical treatment of hyperhidrosis [4]. Surgical treatment by open method is replaced by minimally invasive vidio assisted thoracoscopic sympathectomy. In present days, thoracoscopic sympathectomy is performed by minimally invasive video-thoracoscopy; there are no arguments to justify any open surgery to address the thoracic sympathetic trunk. The sympathetic chain runs under the parietal pleura along each side of the spine, at the level of the necks of the ribs, near the articulation between ribs and vertebrae. The chain is a whitish cord, visible and palpable in the majority of individuals. Even though excessive local fat can preclude a clear identification of the chain, the ribs are usually easy to visualize. Bilateral thoracoscopic sympathectomy is a suitable method of treatment for severe palmar hyperhidrosis but emphasizes the need to offer the patient more information, especially regarding compensatory sweating which seems inescapable [5]. Bilateral thoracoscopic sympathectomy and its variations have been performed in thoracic surgery for more than 100 years. However, its indications have undergone profound modifications in this period. Likewise, since then the surgical technique has also 
evolved dramatically up to the minimally invasive techniques worldwide accessible in present days. The main indication for thoracoscopic sympathectomy is primary hyperhidrosis. This operation has a good result and helps patients to improve their quality of life.

\section{Methods}

A prospective cohort study was made for patients who underwent bilateral thoracoscopic sympathectomy and 36 patients were admitted to the general surgical department, in Al-Kuwait University Teaching Hospital and Al-Thowra Modern University Hospital in Sana'a Capital of YEMEN. Patients underwent thoracoscopic sympathectomy during the study period, which was from January 2017 to May 2020. A chest X-ray was performed the first day after surgery by 6 hours to exclude the possibility of pneumothorax, hemothorax or pulmonary atelectasis. The patient can discharge after 24 - 48 hours post operation.

\subsection{Surgical Methods}

The double-lumen endotracheal intubation was used for general anesthesia, and patients' heart rate, blood pressure, and oxygen saturation were monitored during surgery. The position of patients was $30^{\circ}-45^{\circ}$ half sitting, with arm overstretch. Two small incisions of about $0.5 \mathrm{~cm}$ were made, one in the $3^{\text {rd }}$ intercostal space of the anterior axillary line and the other in the $5^{\text {th }}$ intercostal space of the mid-clavicular line [5]. The thoracoscope is introduced through a port placed in the mid-clavicular line in the fifth intercostal space, the introduced the camera with zero degree angle lens. $\mathrm{CO}_{2}$ is insufflated to $8-10 \mathrm{cmH}_{2} \mathrm{O}$ of pressure to collapse the dome of the lung. The thoracic chain is readily identified covered by the thin layer of the parietal pleura. A diathermy hook is inserted through the operating channel of the thoracoscope. The sympathetic chain is visualized behind the parietal pleura, which is then scored on either side using the cautery to delineate the position of the chain and the extent of the planned cauterization which corresponds to the extent of the chain destroyed. Using the ribs for reference, the sympathetic chain is then cauterized and divided from T2 to T3 for patients with predominantly palmar hyperhidrosis and from $\mathrm{T} 2$ to $\mathrm{T} 4$ for patients with predominantly axillary hyperhidrosis (can be extended to T5 to treat hyperhidrosis of the sole). Electrocautery ablation of the accessory branches and Kuntz nerve, when present, was performed in all cases to prevent relapse of the health status immediately before and six months after surgery [6]. The thoracoscope is then removed and replaced with a small red rubber catheter. With positive pressure ventilation, the catheter is then removed under suction to allow expansion of the lung [7]. The catheter was inserted and then pulled out after complete re-expansion of the lung, followed by a suturing of the incisions. The same procedure was performed on the other side. A chest X-ray was performed the first day after surgery by 6 hours to exclude the possibility of pneumothorax, hemothorax or pulmonary atelectasis [5]. The patient can discharge after $24-48$ 
hours post operation.

\subsection{Exclusion}

Patient sweating was caused by metabolic diseases such as hyperthyroidism, hyper metabolism, tuberculosis, and other systematic diseases. Any case does other level of laparoscopic sympathectomy like lumber sympathectomy. Also any patient who hadn't done previous thoracotomy.

Data collection: Patient's data were collected by filling out a questionnaire from the patient's file, and each patient was sent a questionnaire regarding success of the procedure, and compensatory sweating through their phone number by what App.

Data analysis: The success rate of bilateral thoracoscopic sympathectomy on hyperhidrosis patients, complicated cases and non-complicated cases.

The statistical data analysis will be done by computer using SPSS software program.

Ethical considerations: Approval of study and took a consent from patient about using their medical information.

\section{Results}

Bilateral thoracoscopic sympathectomy was performed in 36 patients. The patients included $75 \%$ males, $25 \%$ females. In addition, the age range was 14 to 32 years old at the time of surgery, most of our patients were between 20 to 29 years old at time of surgery, as shown in Figure 1.

According to our study, we found $100 \%$ of sample complaining of palm hyperhidrosis, some of them associated with sole hyperhidrosis (58.3\%), axilla and sole hyperhydrosis (41.7\%). While $25 \%$ of patients had craniofacial symptoms like facial blushing, as shown in Figure 2.

All patients suffer from primary hyperhidrosis for a long time as childhood (94.5\%), adolescent (5.5\%), and have poor response to drug therapy, so they were advised to surgery.

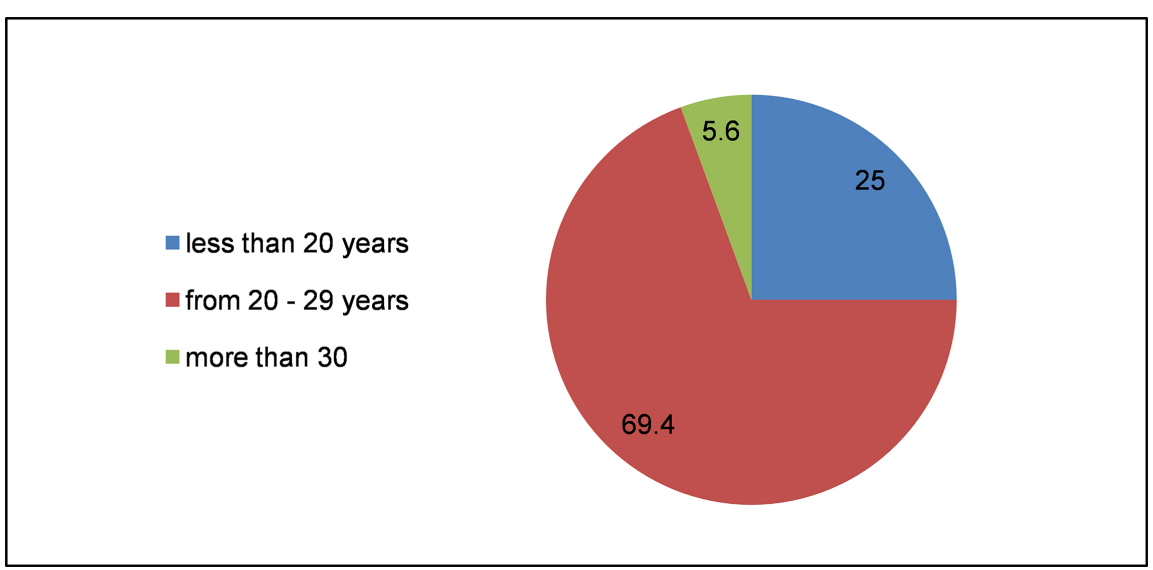

Figure 1. Distribution of patients by age groups. 


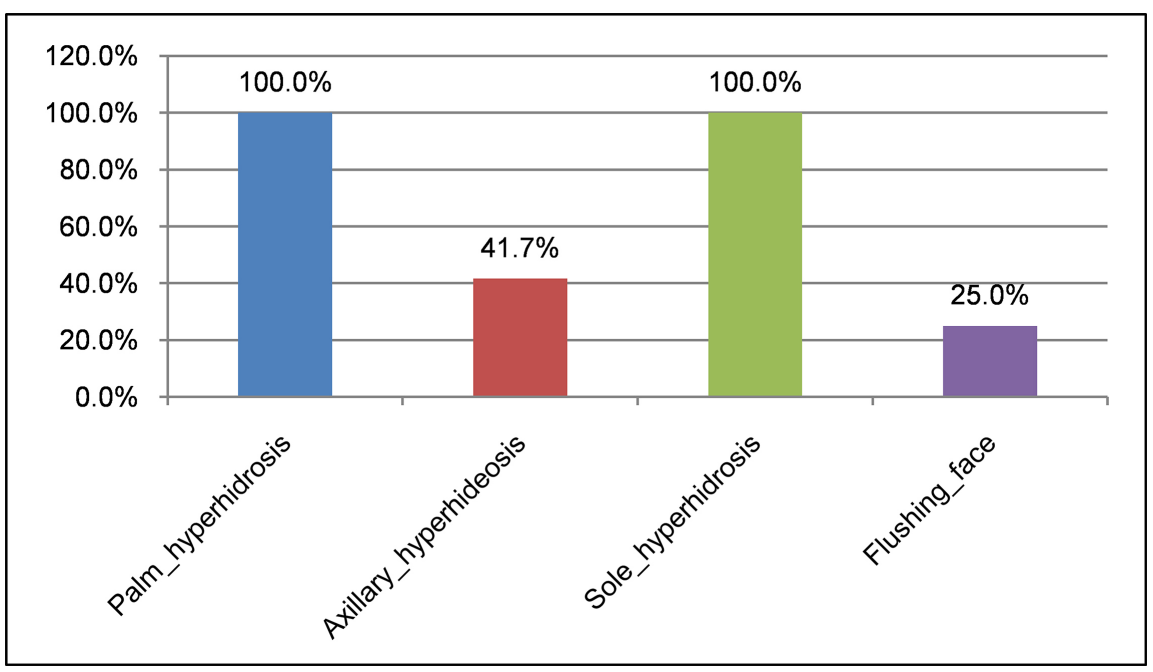

Figure 2. Distribution of patients by sit of excessive sweating.

In this study, we found the surgical technique and level of sympathectomy different according to the site of excessive sweating. Some patients underwent sympathectomy for T2 and T3 only especially in-patient who complains of palm hyperhidrosis without axilla hyperhidrosis, but if there was axillary hyperhidrosis the surgeon preferred to extend to T4 and T5. We found most of the patients had been done sympathectomy at $\mathrm{T} 2, \mathrm{~T} 3$ and $\mathrm{T} 4(66.7 \%)$, but (25\%) at $\mathrm{T} 2, \mathrm{~T} 3$, $\mathrm{T} 4$ and $\mathrm{T} 5$, and (8.3\%) only cauterized $\mathrm{T} 2$ and $\mathrm{T} 3$ as shown in Figure 3.

The success rate post-bilateral thoracoscopic sympathectomy was good; all patients (100\%) improved from hyperhidrosis in palm, (94.4\%) improved from excessive seating in sole and (100\%) improved from axillary hyperhidrosis or facial blushing as shown in Table 1.

\section{Discussion}

Hyperhidrosis is defined as sweating that disrupts normal activities. Episodes of excessive sweating occur at least once a week for no clear reason and have an effect on social life or daily activities. According to the International Hyperhidrosis Association, approximately 2.8 percent of Americans are affected by hyperhidrosis; that's around 7.8 million peoples [8]. For some, hyperhidrosis symptoms are so severe that it becomes embarrassing, causing discomfort and anxiety. The patient's career choices, free time activities, personal relationships, self-image, and emotional well-being may be affected [7]. In our study $22-29$ years old (69.4\%) period of age group, the patients whose more suffering from this problem (male $>$ female), due to it is necessary to interact with society, and This age group is the beginning of public relations, so hyperhidrosis affected their social life and daily activities as shown in Figure 1.

Although its pathophysiology is unclear, it is believed to be caused by hyper-stimulation or over-activity of the sympathetic nervous system that passes through the upper thoracic ganglia. The diagnosis of primary hyperhidrosis is 
based on symptoms, and supported by a specific clinical history. Help is needed to distinguish focal from generalized hyperhidrosis. The Multi-Specialty Working Group on Hyperhydrosis in the United States has proposed some criteria for the diagnosis of focal hyperhidrosis [3] [7]. We found significant improvement in symptoms of hyperhidrosis in the pre-operatively affected areas, all patients were complaining of palm or axillary hyperhidrosis, they improved immediately post operation, whereas patients complaining of sole hyperhidrosis most of them improved (94.4\%), while (5.4\%) not improved. Also patients those complaining of facial blushing pre-operatively improved after the operation (100\%) as shown in Figure 3, Table 1. We think bilateral thoracoscopic sympathectomy for management of hyperhidrosis has a good result. In addition, it demonstrates that compensatory sweating is an almost unavoidable phenomenon post-operative and has related to level of sympathectomy, but this squeal infrequently improves with time.

Other study by Drott et al., reported the success rate was $98.6 \%$ on 850 patients treated by thoracoscopic sympathectomy. While in other study by T. S. Lin et al. published the results of sympathectomy performed on 2200 patients, reporting a successful treatment was $99 \%$ [9]. In additional study for 335 patients with severe primary hyperhidrosis by Murat Öncel et al., most of the patients presented with an improvement in primary hyperhidrosis. The initial cure rate was $95 \%$, the axillary region in 42 (12.53\%), and in the palmar and pedal regions in $22(6.56 \%)$ patients [7].

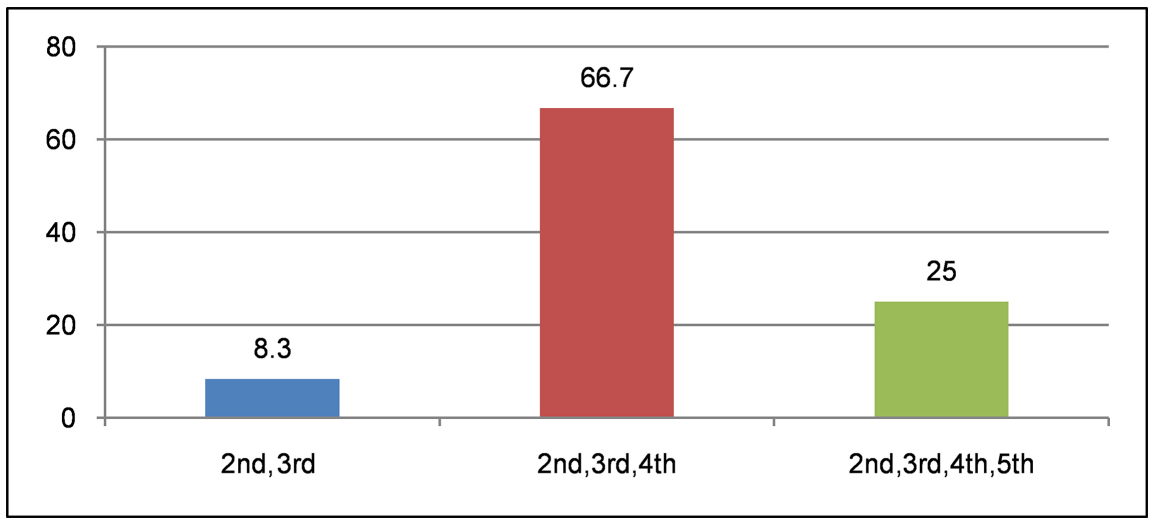

Figure 3. Distribution of patients by the level of sympathectomy.

Table 1. Distribution of site of sweating and their improvement post-operative.

\begin{tabular}{ccccc}
\hline Site of sweating & No of patient & Percentage & Improve & Not improve \\
\hline Palm & 36 & $100 \%$ & $100 \%$ & $0 \%$ \\
Sole & 36 & $100 \%$ & $94.4 \%$ & $5.6 \%$ \\
Axilla & 12 & $33.3 \%$ & $100 \%$ & $0 \%$ \\
Facial blushing & 9 & $25 \%$ & $100 \%$ & $0 \%$ \\
\hline
\end{tabular}


Neelan Doolabh et al. study, they found the success rates were palmar 100\% (109/109), axillary 98\% (48/49), and face/scalp 93\% (26/28). Plantar hyperhidrosis responded with improvement in $82 \%(72 / 88)$ of all patients [10]. So according to all mentioned above studies and to our study, bilateral thoracoscopic sympathectomy has a good result and high success rate.

In our study, we observed all patients improved from hyperhidrosis in palm, and some of patients improved in other site especially if sympathectomy level was at T4 and T5, like in axilla the improvement was $33.3 \%$, and in sole the improvement was $94.4 \%$ when the sympathectomy level reach to T4 and T5. Only two cases were not improved from sole hyperhidrosis where the sympathectomy was T2 and T3 only as shown in Figure $3 \&$ Table 1.

In Murat Öncel study, T2 resection was performed in 281 patients with craniofacial hyperhidrosis, T3 resection was performed in 94 patients with only palmar and only axillary hyperhidrosis, T3-T4 resection was performed in 175 patients with palmar and axillary hyperhyidrosis, and T3-T5 resection was performed in 22 patients with palmar and pedal hyperhidrosis, the initial cure rate was $95 \%$ [7]

According to our data after operation, only two cases (5.6\%) developed pneumothorax and they need chest drainage for 24 hours then discharge, and 6 cases $(16.7 \%)$ complained of severe pain at site of operation and upper limb, this pain improved with time. No other early post operation complication or wound complication. No cases of persistent hyperhidrosis were observed after discharge as shown in Table 2.

In previous study by Pedro M. Rodríguez et al., there were complications in 23 patients (5.6\%) consisting of: 16 cases of pneumothorax (3.9\%) in which 11 were less than $20 \%$ and did not require pleural drainage, 2 cases of unilateral and transitory Horner syndrome (0.5\%), 2 cases of hemothorax requiring pleural drainage (2.5\%), 1 case of quilothorax (0.25\%) treated with video-assisted thoracic surgery, 1 case of cubital nerve neuropraxia $(0.25 \%)$ from which the patient recovered in 21 days, and 1 case of subcutaneous cervico-thoracic emphysema (0.25\%) [11].

Compensatory hyperhidrosis one of the main late post-operative complication occurred in half of patients (50\%) in our study, 33\% in back and chest and $17 \%$ in back and abdomen. The post-operative complication can be divided into early and

Table 2. Post-operative complications, $\mathrm{CH}$ : compensatory hyperhidrosis.

\begin{tabular}{cccc}
\hline \multicolumn{2}{c}{ Post-operative complication } & NO & Percentage \\
\hline Early post-operative complication & Pneumothorax & 2 & $5.6 \%$ \\
Late post-operative complication & Severe pain & 6 & $16.7 \%$ \\
No complications & $\mathrm{CH}$ in trunk & 18 & $50 \%$ \\
\hline
\end{tabular}


late. Early post-operative complications were two cases complicated by pneumothorax (5.6\%), and six cases suffering from severe pain at site of operation (16.7\%). Late post-operative complications were 18 cases whose represented 50\% of patients, they complicated by compensatory hyperhidrosis in the trunk, as shown in Table 2. Other study by Z. Steiner et al has indicated a more variable response. Steiner et al. report immediate development of compensatory hyperhidrosis in half of respondents [12]. There is a relationship between level of sympathectomy and rate of compensatory hyperhidrosis. If we got level of sympathectomy low, the rate of sympathectomy increased. However, the highest rate (40.5\%) of compensatory hyperhidrosis was found in cauterization $2^{\text {nd }}, 3^{\text {rd }}$ and $4^{\text {th }}$ ganglions, because most of the cases were at this level. More extensive sympathectomy to the level of $\mathrm{T} 4$ or $\mathrm{T} 5$ is associated with increased compensatory hyperhidrosis rate, because during operation, there are more manipulation and more injury for sympathetic nerves as shown in Table 3 and Figure 4.

In Peter B. Licht et al. study, they found compensatory sweating occurred in 131 patients $(89 \%)$, and was located on the abdomen in 83 patients $(63 \%)$, the back in 90 patients (69\%), the lower extremities in 56 patients $(43 \%)$, and the chest in 26 patients (20\%) [13].

Table 3. Distribution of the post-operative complication.

\begin{tabular}{cccc}
\hline Site of sympathectomy & $\begin{array}{c}\text { NO of patient according } \\
\text { to level sympathectomy }\end{array}$ & $\begin{array}{c}\text { NO of patient } \\
\text { complicated by CH }\end{array}$ & Percentage \\
\hline $\mathbf{2}^{\text {nd }}, \mathbf{3}^{\text {rd }}$ & 3 & 0 & $0 \%$ \\
$\mathbf{2}^{\text {nd }}, \mathbf{3}^{\text {rd }}, \mathbf{4}^{\text {th }}$ & 24 & 15 & $40.5 \%$ \\
$\mathbf{2}^{\text {nd }}, \mathbf{3}^{\text {rd }}, \mathbf{4}^{\text {th }}, \mathbf{5}^{\text {th }}$ & 9 & 3 & $8.1 \%$ \\
Total & 36 & 18 & $50 \%$ \\
\hline
\end{tabular}

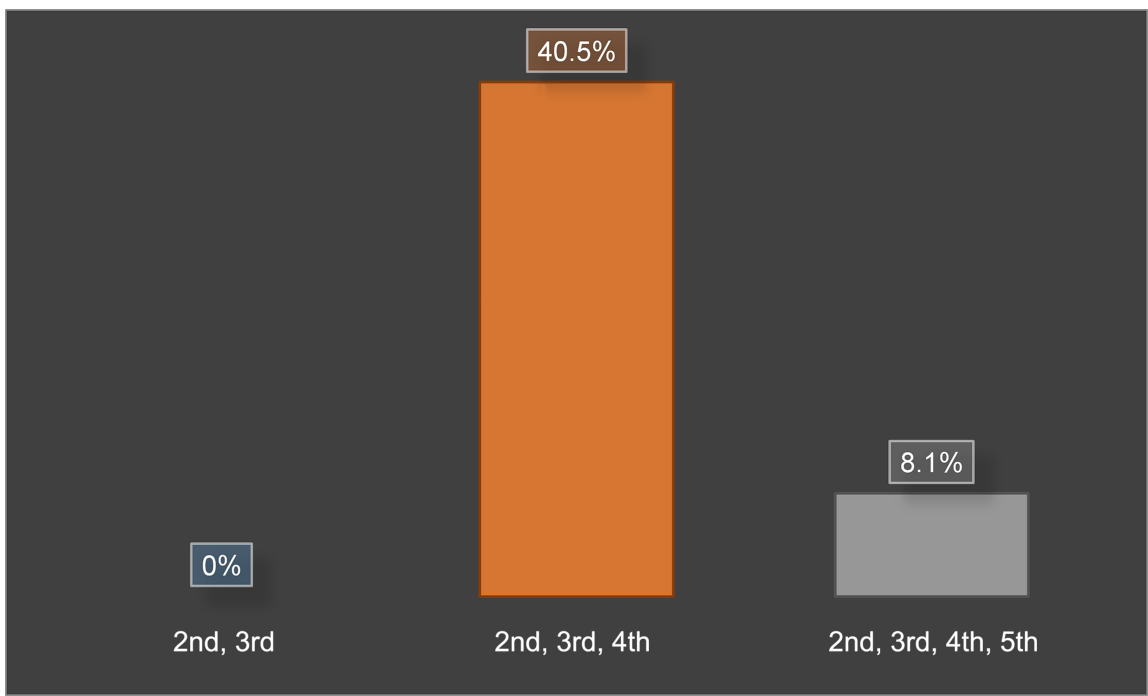

Figure 4. Relationship between level of sympathectomy and rate of compensatory hyperhidrosis. 
In C S Cinà et al. study, of the 22 patients who underwent surgery, one patient (5\%) developed a postoperative pneumothorax requiring placement of a Heimlich valve. There were no other local or wound complications. No patient developed Horner's syndrome or any other neurologic complication [6]. In study by M. Riet, et al., 28-thoracoscopic sympathectomy is for palmar and/or axillary hyperhidrosis. In all patients, the sympathetic chain was transected cranially and caudally to the third ganglion. The surgery was effective in all patients. After a median follow-up of 3.5 years, compensatory hyperhidrosis was not recorded in any of the patients [14]. We suggest that a more extensive sympathectomy to the level of T4 or T5 is associated with increased compensatory hyperhidrosis rate, because during operation, there more manipulation and injury more for sympathetic nerves. Mortality rate post-bilateral thoracoscopic sympathectomy is almost zero, so thoracoscopic sympathectomy is less invasive and safe.

\section{Conclusions}

- Thoracic sympathectomy and its variations are the most valuable tools in the curative treatment for hyperhidrosis.

- Those presenting with palmar hyperhidrosis are the most suitable for surgery as first-line treatment.

- For patients with axilla, sole and/or facial complain, sympathectomy gives good results.

- The surgery is performed by means of minimally invasive approaches and the complication's rates are very low.

- Nevertheless, the occurrence of compensatory hyperhidrosis should never be ignored and all maneuvers to prevent its occurrence.

\section{Recommendation}

- Thoracoscopic sympathectomy is the best option for treated hyperhidrosis after failed medical management, to improve quality of patient life.

- Thoracoscopic sympathectomy is low invasive operation, nearly no mortality rate and has good outcomes for patients.

- Should inform the patient about post-operative complications especially compensatory hyperhidrosis.

\section{Conflicts of Interest}

The authors declare no conflicts of interest regarding the publication of this paper.

\section{References}

[1] Sammons, J.E. and Khachemoune, A. (2017) Axillary Hyperhidrosis: A Focused Review. Journal of Dermatological Treatment, 28, 582-590. https://doi.org/10.1080/09546634.2017.1309347

[2] Romero, F.R., Haddad, G.R., Miot, H.A. and Cataneo, D. (2016) Palmar Hyperhidrosis: Clinical, Pathophysiological, Diagnostic and Therapeutic Aspects. Anais Brasileiros de Dermatologia, 91, 716-725. 
https://doi.org/10.1590/abd1806-4841.20165358

[3] Vannucci, F. and Araújo, J.A. (2017) Thoracic Sympathectomy for Hyperhidrosis: From Surgical Indications to Clinical Results. Journal of Thoracic Surgery, 9, S178-S192. https://doi.org/10.21037/jtd.2017.04.04

[4] Currie, A.C., Evans, J.R. and Thomas, P.R.S. (2011) An Analysis of the Natural Course of Compensatory Sweating Following Thoracoscopic Sympathectomy. International Journal of Thoracic Surgery, 9, 437-439. https://doi.org/10.1016/j.ijsu.2011.04.006

[5] Dumont, P., et al. (2004) Long-Term Results of Thoracoscopic Sympathectomy for Hyperhidrosis. Annals of Thoracic Surgery, 78, 1801-1807.

https://doi.org/10.1016/j.athoracsur.2004.03.012

[6] Prasad, A. (2008) Endoscopic Thoracic Sympathectomy for Hyperhidrosis: Technique and Results. Journal of Minimal Access Surgery, 4, 23. https://doi.org/10.4103/0972-9941.40995

[7] Öncel, M., Sunam, G.S., Erdem, E., Dereli, Y., Tezcan, B. and Akyol, K.G. (2013) Bilateral Thoracoscopic Sympathectomy for Primary Hyperhydrosis. Cardiovascular Journal of Africa, 24, 137-140. https://doi.org/10.5830/CVJA-2013-007

[8] Lin, T.S., Wang, N.P. and Huang, L.C. (2001) Pitfalls and Complication Avoidance Associated with Transthoracic Endoscopic Sympathectomy for Primary Hyperhidrosis (Analysis of 2200 Cases). International Journal of Surgical Investigation, 2, 377-385.

[9] Doolabh, N., Horswell, S., Williams, M., et al. (2004) Thoracoscopic Sympathectomy for Hyperhidrosis: Indications and Results. The Annals of Thoracic Surgery, 77, 410-414. https://doi.org/10.1016/j.athoracsur.2003.06.003

[10] Deng, B., Tan, Q.-Y., Jiang, Y.-G., Zhao, Y.-P., Zhou, J.-H., Ma, Z. and Wang, R.-W. (2010) Optimization of Sympathectomy to Treat Palmar Hyperhidrosis: The Systematic Review and Meta-Analysis of Studies Published during the Past Decade. Surgical Endoscopy, 25, 1893-1901. https://doi.org/10.1007/s00464-010-1482-3

[11] Hashmonai, M., Cameron, A.E.P., Licht, P.B., Hensman, C. and Schick, C.H. (2016) Thoracic Sympathectomy: A Review of Current Indications. Surgical Endoscopy, 30, 1255-1269. https://doi.org/10.1007/s00464-015-4353-0

[12] Rodríguez, P.M., Freixinet, J.L., Hussein, M., Valencia, J.M., Gil, R.M., Herrero, J. and Caballero-Hidalgo, A. (2008) Side Effects, Complications and Outcome of Thoracoscopic Sympathectomy for Palmar and Axillary Hyperhidrosis in $406 \mathrm{~Pa}$ tients. European Journal of Cardio-Thoracic Surgery, 34, 514-519.

https://doi.org/10.1016/j.ejcts.2008.05.036

[13] Licht, P.B. and Pilegaard, H.K. (2004) Severity of Compensatory Sweating after Thoracoscopic Sympathectomy. The Annals of Thoracic Surgery, 78, 427-431. https://doi.org/10.1016/j.athoracsur.2004.02.087

[14] Riet, M., Smet, A.A.E.A., Kuiken, H., Kazemier, G. and Bonjer, H.J. (2001) Prevention of Compensatory Hyperhidrosis after Thoracoscopic Sympathectomy for Hyperhidrosis. Surgical Endoscopy, 15, 1159-1162.

https://doi.org/10.1007/s004640090097 